Ciencia y Salud, Vol. IV, No. 3, septiembre-diciembre, 2020 • ISSN (impreso): 2613-8816• ISSN (en línea): 2613-8824

DOI: https://doi.org/10.22206/cysa.2020.v4i3.pp53-61

\title{
SEPSIS EN PACIENTES CON TUMORES SÓLIDOS EN QUIMIOTERAPIA
}

\section{Sepsis in patients with solid tumors in chemotherapy}

\section{Mónica Lisandra Carvajal Aballe ${ }^{a}$ Iraida Aguirrechu Caballero ${ }^{b}$}

Recibido: 14 de marzo, 2020 • Aprobado: 27 de mayo, 2020

Cómo citar: Carvajal Aballe M, Aguirrechu Caballero I. Sepsis en pacientes con tumores sólidos en quimioterapia. cysa [Internet]. 18 de septiembre de 2020 [citado 18 de septiembre de 2020];4(3):53-61. Disponible en: https://revistas.intec.edu.do/index.php/cisa/ article/view/1919

\section{Resumen}

El cáncer constituye un problema de salud mundial. Múltiples factores relacionados con la enfermedad y los tratamientos provocan predisposición a las infecciones en el paciente oncológico. La sepsis, constituye una importante causa de morbimortalidad. Se realizó un estudio descriptivo de corte transversal, con el objetivo de describir el comportamiento de las infecciones en pacientes con tumores sólidos en quimioterapia, atendidos en el servicio de Oncología Médica del Hospital Clínico Quirúrgico Hermanos Ameijeiras, de enero 2015 a enero 2017. La media de edad fue 53 años $(32,3 \%)$ y predominaron los pacientes masculinos $(56.5 \%)$. Los tumores de vías digestivas, carcinomas de pulmón y sarcomas de partes blandas representaron el 32.3, 25 y $13.7 \%$, respectivamente. De los 124 eventos de sepsis reportados, el $39.5 \%$ fueron neutropenia febril, seguido por sepsis urinaria $(34.7 \%)$. En pacientes con otras infecciones, predominaron la nuemopatía inflamatoria y la mucositis grado IV. No existió asociación estadística significativa entre el esquema de quimioterapia y la

\footnotetext{
a Especialista de Primer Grado en Oncología Médica, Especialista de Primer Grado en Medicina General Integral, Máster en Enfermedades Infecciosas, Instituto Nacional de Oncología y Radiobiología, La Habana, Cuba. ORCID: 0000-0002-1453-0526
}

\begin{abstract}
Cancer is a global health problem. Multiple factors related to the disease and treatments cause a special predisposition to infections in the cancer patient. Sepsis is an important cause of morbidity and mortality. A descriptive cross-sectional study was carried out, where all sepsis events confirmed by microbiology were included, in patients with solid tumors in chemotherapy, treated at the Medical Oncology service of the Hospital Clinico Quirúrgico Hermanos Ameijeiras, from January 2015 to January 2017, whose objective was to describe their behavior. The mean age was 53 years $(32.3 \%)$ and male patients predominated $(56.5 \%)$. Digestive tract tumors, lung carcinomas, and soft tissue sarcomas accounted for 32.3, 25 , and $13.7 \%$, respectively. Of the 124 sepsis events, $39.5 \%$ had febrile neutropenia, followed by urinary sepsis (34.7\%). In the group of patients with other infections, inflammatory novel lung disease predominated, followed by grade IV mucositis. There was no significant statistical association between the chemotherapy schedule and the

\footnotetext{
b Especialista de Segundo Grado en Oncología Médica. Profesora Auxiliar. Máster en Enfermedades Infecciosas. Hospital Clínico Quirúrgico Hermanos Ameijeiras. La Habana, Cuba.

ORCID: 0000-0002-1044-6052
} 
aparición de infección. El avance y perfeccionamiento de los tratamientos han prolongado la vida de los pacientes y esto ha favorecido el mejor estudio y compresión de los diferentes eventos adversos, relacionados con la administración de quimioterapia.

Palabras clave: paciente oncológico; sepsis; tumor sólido; quimioterapia.

\section{Introducción}

El perfeccionamiento del tratamiento, el avance en los diagnósticos y el conocimiento de nuevos fármacos han prolongado la vida del paciente oncológico; sin embargo, esto también se relaciona con la aparición de eventos adversos. La sepsis en pacientes oncológicos, principalmente en quimioterapia, constituye una causa importante de morbimortalidad. La neutropenia febril es una urgencia oncológica, que puede ser fatal si no se inicia antibioticoterapia precoz. ${ }^{1}$

Los pacientes oncológicos en quimioterapia presentan alteraciones de uno o más de los mecanismos de defensa naturales. La piel y las mucosas constituyen la primera barrera frente a las infecciones. Tanto los linfocitos B como los linfocitos $\mathrm{T}$, responsables de la inmunidad humoral y celular, respectivamente, pueden verse afectados. Una disminución en su número y/o función resulta en un incremento severo del riesgo de infecciones. La malnutrición se asocia a alteraciones de la función fagocítica y disminución de la movilidad de los macrófagos. ${ }^{2,3}$ Los tumores sólidos frecuentemente presentan necrosis asociada, lo que favorece la sepsis tumoral. Existen anticuerpos monoclonales que se han asociado con un aumento del riesgo de infecciones oportunistas como el Rituximab; los esteroides también aumentan el riesgo., ${ }^{4,5}$

La hematopoyesis normal, es el resultado del equilibrio entre la producción de células sanguíneas, su appearance of infection. The advancement and improvement of the treatments have prolonged the life of the patients and this has favored the better study and understanding of the different adverse events related to the administration of chemotherapy.

Keywords: oncological patient; sepsis; solid tumor; chemotherapy.

supervivencia en el sistema vascular y eliminación. Los fármacos antineoplásicos inducen mecanismos de apoptosis mediante daños provocados a su ácido desoxirribonucleico, así como inhibición del crecimiento celular, ejerciendo su acción en células tumorales y también sobre tejidos sanos. ${ }^{6,7}$ Existen infecciones oportunistas como son las micóticas, siendo la Cándida albicans el gérmen más frecuente. La candidiasis se presenta de forma frecuente como placas adheridas a la mucosa. Las infecciones de catéter vasculares utilizados para administrar el tratamiento, también constituyen una de las puertas de entrada para microorganismos, fundamentalmente de Staphylococcus y Streptococus. Es imprescindible descartar una bacteriemia en un paciente con quimioterapia y síndrome febril, mediante la realización de hemocultivos. ${ }^{8,9}$

Las infecciones virales predominantes en los pacientes oncológicos son producidas por Herpes simple, Herpes zoster y Citomegalovirus. Es muy elevada la frecuencia de estomatitis herpética tras mucositis en pacientes que reciben quimioterapia, con presencia de úlceras dolorosas en la mucosa oral. La reactivación de Herpes zoster es frecuente en estos pacientes. ${ }^{10}$

La sepsis respiratoria suele presentarse en pacientes con carcinoma de pulmón como una neumonitis sobreinfestada. Los pacientes con patologías crónicas respiratorias, también son más susceptibles de presentar infecciones respiratorias bajas. Los virus respiratorios más frecuentes son la

54 | Ciencia y Salud 2020; 4(3, septiembre-diciembre): Casos clínicos • 53-61 
influenza, parainfluenza, sincitial virus respiratorio $y$ adenovirus, teniendo un comportamiento clínico semejante a la población en general. Los infiltrados pulmonares se deben diferenciar también de fibrosis radiógena o toxicidades propias de algunos fármacos citostáticos como la bleomicina. ${ }^{11,12}$

Los pacientes oncológicos con cirugías intraabdominales, fundamentalmente de colon y vías biliares, pueden presentar complicaciones propias del tratamiento quirúrgico que pueden aparecer en el período del tratamiento con quimioterapia, tal es el caso de los abscesos intraabdominales y fístulas sobreinfestadas. ${ }^{13}$

La función renal se puede ver afectada por una reducción en la ingesta de líquidos (mucositis, disfagia) o bien por incremento en las pérdidas (emesis, diarreas, hemorragias). En ocasiones disminuye la perfusión renal o se produce una mala distribución de fluidos (insuficiencia cardíaca congestiva, edemas, ascitis). Otras veces se compromete la función por el depósito de elementos propios del organismo (hipercalcemia) o por infiltración u obstrucción tumoral. ${ }^{14,15}$

El hemocultivo es la herramienta diagnóstica más utilizada para determinar el agente etiológico, si se sospecha una bacteriemia. Las guías de la IDSA (Infectiuos Diseasis Society of America por sus siglas en inglés) recomiendan realizarlos en la valoración inicial de pacientes con neutropenia febril. ${ }^{16-18}$ Los costos que acarrean estos pacientes al sistema de salud son elevados en términos de hospitalizaciones y antibioticoterapia. Así como incumplimientos de los esquemas de quimioterapia con la consecuente disminución de su eficacia terapéutica. ${ }^{19-21}$

A pesar de los avances en el conocimiento de los factores de riesgo, del diagnóstico y tratamiento, las infecciones continúan siendo una causa frecuente de morbimortalidad en los pacientes oncológicos. ${ }^{22}$ Los estándares de manejo de las enfermedades infecciosas en pacientes con tumores sólidos en quimioterapia varían de acuerdo al mapa microbiológico de cada institución de salud; de ahí la importancia de realizar este tipo de investigación descriptiva, con el fin de poder actualizar los protocolos de actuación de cada centro hospitalario.

\section{Material y método}

Se realizó un estudio descriptivo y transversal, con el propósito de describir el comportamiento de la sepsis asociada a quimioterapia en pacientes con tumores sólidos, atendidos en el servicio de Oncología del Hospital Hermanos Ameijeiras de enero 2015 a enero 2017. Se incluyeron todos los pacientes con tumores sólidos en quimioterapia y sepsis asociada, que cumplieron los criterios de selección: pacientes con edad mayor o igual a 18 años, que desarrollaron algún episodio séptico febril en el período de la quimioterapia, que recibieron antibioticoterapia. Las variables se recolectaron en un instrumento diseńado para este fin y los datos se procesaron en una base de datos en Excel; para el análisis se usó estadística descriptiva. Toda información recolectada se utilizó bajo estricta confidencialidad por parte de los investigadores.

\section{Resultados}

Se presenta la experiencia clínica resultado de una investigación donde se incluyeron un total de 124 episodios de sepsis asociada al tratamiento con quimioterapia. Se analizaron tres subgrupos de eventos, que fueron la neutropenia febril, la sepsis urinaria y otras infecciones como la neumopatía inflamatoria, la mucositis grado IV y otras. La edad media de presentación fue de 53 años y predominó el sexo masculino $(56.5 \%)$. Según el diagnóstico histológico, los tumores de vías digestivas, carcinomas de pulmón y sarcomas de partes blandas representaron el 32.3, 25 y $13.7 \%$, respectivamente, del total de pacientes incluidos en la investigación. (Tabla 1) 
Tabla 1. Distribución de los pacientes según sus características clínicas e histopatológicas $(\mathrm{n}=124)$

\begin{tabular}{|c|c|}
\hline \multicolumn{2}{|c|}{ Características clínico-histopatológicas } \\
\hline \multicolumn{2}{|l|}{ Edad (ańos) } \\
\hline \multicolumn{2}{|c|}{$53,83(15,353)$} \\
\hline \multicolumn{2}{|l|}{ Sexo N (\%) } \\
\hline Femenino & $54(43,5)$ \\
\hline Masculino & $70(56,5)$ \\
\hline \multicolumn{2}{|c|}{$\begin{array}{c}\text { Diagnóstico histológico del tumor } \\
\text { primarioc } \mathrm{N}(\%)\end{array}$} \\
\hline Carcinoma de pulmón & $31(25)$ \\
\hline Sarcoma de partes blandas & $17(13,7)$ \\
\hline Tumor de vías digestivas & $40(32,3)$ \\
\hline Tumores ginecológico & $6(4,8)$ \\
\hline Tumor de células germinales & $12(9,7)$ \\
\hline Carcinoma de mama & $9(7,3)$ \\
\hline Adenocarcinoma de próstata & $5(4,0)$ \\
\hline Adenocarcinoma de páncreas & $4(3,2)$ \\
\hline Total & 124 \\
\hline
\end{tabular}

Fuente: elaboración propia a partir de registros de datos en historias clínicas.

De los 124 eventos que se incluyeron en la investigación, el $39.5 \%$ fueron neutropenia febril, seguido por la sepsis urinaria (34.7\%). De los 49 eventos de neutropenia febril, no se documentó clínicamente foco de infección en 25 pacientes, lo que representó el $51 \%$, existiendo focalización clínica en el $48.9 \%$. En el grupo de pacientes con otras infecciones asociadas, predominó la neumopatía inflamatoria (12.1\%), seguido por la mucositis grado IV. (Tabla 2)
Tabla 2. Distribución de los pacientes con sepsis asociadas a quimioterapia según presentación clínica y por parámetros de laboratorio

\begin{tabular}{|c|c|}
\hline \multicolumn{2}{|c|}{ Tipo de sepsis No (\%) } \\
\hline Neutropenia febril & $\mathbf{4 9}(\mathbf{3 9}, 5)$ \\
\hline Con focalización clínica & $24(48.9)$ \\
\hline Sin focalización clínica & $25(51)$ \\
\hline Sepsis urinaria & $\mathbf{4 3}(\mathbf{3 4 , 7 )}$ \\
\hline Otras sepsis asociadas & $\mathbf{3 2}(\mathbf{2 5 , 8 )}$ \\
\hline Neumopatía inflamatoria & $15(12,1)$ \\
\hline Mucositis Grado IV & $8(6,5)$ \\
\hline Enterocolitis & $2(1,6)$ \\
\hline Absceso cutáneo & $2(1,6)$ \\
\hline Sepsis herida quirúrgica & $2(1,6)$ \\
\hline Sepsis catéter vascular & $1(0,8)$ \\
\hline Absceso intra-abdominal & $2(1,6)$ \\
\hline Total & $124(100)$ \\
\hline
\end{tabular}

Fuente: elaboración propia a partir de registros de datos en historias clínicas.

En el grupo de pacientes que presentaron neutropenia febril, predominó el carcinoma de pulmón, seguidos por los tumores de vías digestivas y sarcomas de partes blandas; representando el 26.5, 22.4 y $20.4 \%$, respectivamente. Del total de pacientes con sepsis urinaria, el 41,9\% tenía tumores de vías digestivas. En el subgrupo que presentó otras sepsis asociadas a la quimioterapia (excluyendo neutropenia febril y sepsis urinaria) predominó el diagnóstico de carcinoma de pulmón. (Tabla 3) 
Tabla 3. Distribución de pacientes según tipo de sepsis y diagnóstico histológico del tumor.

\begin{tabular}{|c|c|c|c|c|c|c|c|c|}
\hline \multirow{3}{*}{$\begin{array}{l}\text { Diagnóstico } \\
\text { histológico del tumor }\end{array}$} & \multicolumn{6}{|c|}{ Tipo de sepsis } & \multirow{2}{*}{\multicolumn{2}{|c|}{ Total }} \\
\hline & \multicolumn{2}{|c|}{ Neutropenia febril } & \multicolumn{2}{|c|}{ Sepsis urinaria } & \multicolumn{2}{|c|}{ Otras } & & \\
\hline & No & $\%$ & No & $\%$ & No & $\%$ & No & $\%$ \\
\hline $\begin{array}{l}\text { Carcinoma de } \\
\text { pulmón }\end{array}$ & 13 & 26,5 & 7 & 16,3 & 11 & 34,4 & 31 & 25,0 \\
\hline $\begin{array}{c}\text { Sarcoma de partes } \\
\text { blandas }\end{array}$ & 10 & 20,4 & 3 & 7,0 & 4 & 12,5 & 17 & 13,7 \\
\hline $\begin{array}{l}\text { Tumor de vías } \\
\text { digestivas }\end{array}$ & 11 & 22,4 & 18 & 41,9 & 11 & 34,4 & 40 & 32,3 \\
\hline Tumor ginecológico & 4 & 8,2 & 2 & 4,7 & 0 & - & 6 & 4,8 \\
\hline $\begin{array}{l}\text { Tumor de células } \\
\text { germinales }\end{array}$ & 4 & 8,2 & 6 & 14,0 & 2 & 6,3 & 12 & 9,7 \\
\hline Carcinoma de mama & 4 & 8,2 & 4 & 9,3 & 1 & 3,1 & 9 & 7,3 \\
\hline $\begin{array}{c}\text { Adenocarcinoma de } \\
\text { próstata }\end{array}$ & 3 & 6,1 & - & - & 2 & 6,3 & 5 & 4,0 \\
\hline $\begin{array}{c}\text { Adenocarcinoma de } \\
\text { páncreas }\end{array}$ & - & - & 3 & 7,0 & 1 & 3,1 & 4 & 3,2 \\
\hline Total & 49 & 100,0 & 43 & 100,0 & 32 & 100,0 & 124 & 100,0 \\
\hline
\end{tabular}

Fuente: elaboración propia a partir de registros de datos en historias clínicas.

En el subgrupo de pacientes con neutropenia febril predominaron aquellos que recibieron quimioterapia con esquemas a base de cisplatino $(32,6 \%)$, seguidos por aquellos regímenes basados en alquilantes del tipo ifosfamida y ciclofosfamida $(24,4$ $\%)$. Los esquemas a base de fluoropirimidinas, fueron los que predominaron en pacientes con infección urinaria $(41,9 \%)$. En cuanto al subgrupo de pacientes que presentaron otras infecciones, predominaron aquellos que recibieron quimioterapia a base de cisplatino $(31,3 \%)$ y fluoropirimidinas $(28,1 \%)$. (Tabla 4$)$ 
Tabla 4. Distribución de pacientes según tipo de sepsis y esquemas de quimioterapia recibida

\begin{tabular}{|c|c|c|c|c|c|c|c|c|}
\hline \multirow{3}{*}{ Esquemas de quimioterapia } & \multicolumn{6}{|c|}{ Tipo de sepsis } & \multirow{2}{*}{\multicolumn{2}{|c|}{ Total }} \\
\hline & \multicolumn{2}{|c|}{$\begin{array}{l}\text { Neutropenia } \\
\text { febril }\end{array}$} & \multicolumn{2}{|c|}{$\begin{array}{l}\text { Sepsis } \\
\text { urinaria }\end{array}$} & \multicolumn{2}{|c|}{ Otras sepsis } & & \\
\hline & No & $\%$ & No & $\%$ & No & $\%$ & No & $\%$ \\
\hline A base de cisplatino & 16 & 32,6 & 9 & 20,9 & 10 & 31,3 & 35 & 28,2 \\
\hline A base fluoropirimidina & 8 & 16,3 & 18 & 41,9 & 9 & 28,1 & 35 & 28,2 \\
\hline A base carboplatino & 6 & 12,2 & 5 & 11,6 & 4 & 12,5 & 15 & 12,1 \\
\hline $\mathrm{AC} / \mathrm{DC}$ & 2 & 4,1 & 3 & 7,0 & 1 & 3,1 & 6 & 4,8 \\
\hline Esquema con alquilantes & 12 & 24,4 & 4 & 9,3 & 5 & 15,6 & 21 & 16,9 \\
\hline A base gemcitabina & 1 & 2,0 & 4 & 9,3 & 1 & 3,1 & 6 & 4,8 \\
\hline Docetaxel/prednisona & 3 & 6,1 & 0 & 0,0 & 2 & 6,3 & 5 & 4,0 \\
\hline Vincristina & 1 & 2 & 0 & 0,0 & 0 & 0,0 & 1 & 0,8 \\
\hline Total & 49 & 100 & 43 & 100,0 & 32 & 100,0 & 124 & 100,0 \\
\hline
\end{tabular}

Fuente: elaboración propia a partir de registros de datos en historias clínicas.

\section{Discusión}

El conocimiento de las infecciones asociadas a la quimioterapia en pacientes con tumores sólidos y su correcto manejo terapéutico son determinantes para reducir la elevada morbimortalidad asociada. La neutropenia febril es una entidad clínica heterogénea que incluye pacientes con riesgos muy variables de desarrollar complicaciones médicas graves, lo que puede provocar pérdida en la intensidad de dosis del tratamiento, disminuyendo así la eficacia terapéutica.

Los pacientes mayores de 65 años generalmente presentan un elevado riesgo de complicaciones durante un episodio de infección durante la quimio- terapia; pues este grupo de pacientes generalmente presentan una reserva medular baja, malnutrición asociada o alguna patología orgánica concomitante.

La edad media de presentación de la sepsis asociada a la quimioterapia está influenciada por varios factores tales como la edad de presentación promedio de las enfermedades oncológicas en los diferentes países, la prevalencia de la patología en cuestión, así como la supervivencia medida en años, pues el cáncer constituye una enfermedad crónica y según avanzan las tecnologías y la adquisición de nuevos fármacos también se va modificando el panorama de la enfermedad en cada país. ${ }^{23}$ En una investigación prospectiva donde se analizaron 579 episodios de bacteriemia en pacientes con

58 | Ciencia y Salud 2020; 4(3, septiembre-diciembre): Casos clínicos • 53-61 
neutropenia febril Marín et al., observaron que la neutropenia febril, fue ocho veces más frecuente en pacientes con enfermedades oncohematológicas en comparación con pacientes con tumores sólidos. ${ }^{24}$

Con frecuencia no se logra documentar la etiología de la enfermedad infecciosa durante el episodio febril, esto solo ocurre entre el $20 \%$ y el $30 \%$ de los casos; ${ }^{25}$ los resultados obtenidos en la presente investigación sobrepasan estos valores (51\%). Santo et al., en una investigación donde incluyeron 70.662 pacientes oncológicos, constataron que la herida quirúrgica fue el sitio de focalización clínica de la sepsis más frecuente $(26,11 \%)$, seguido por la bacteriemia $(24,11 \%)$ y la sepsis respiratoria $(18,50 \%){ }^{1}$ estos datos coinciden con la serie de pacientes oncológicos que estudió Velázquez et al., en el Instituto Jalisciense de Cancerología, México, donde predominó la infección de la herida quirúrgica (27\%), seguido por las neumonías intrahospitalaria y las infección de vías urinarias. ${ }^{26}$

La correlación entre la neutropenia febril y la enfermedad oncológica de base está determinada fundamentalmente por los esquemas de quimioterapia que se administraron según los protocolos de tratamiento. En la casuística actual influye la elevada prevalencia del cáncer de pulmón en la población cubana, por tanto, es lógico encontrar que estos pacientes predominaron al determinar el comportamiento de la neutropenia febril. Las infecciones del tracto urinario son muy frecuentes como consecuencia de la utilización de dispositivos en la vía urinaria como sondas; existencia de inmunosupresión, aparición de obstrucción urinaria debida al propio tumor, por la presencia de distorsiones de la vía urinaria por antecedentes quirúrgicos (realización de derivaciones urinarias) o por invasión directa por el tumor. Los tumores intraabdominales pueden producir compresión o infiltración del tracto urinario, lo cual favorece el desarrollo de infecciones urinarias a repetición y muchas veces refractarias al tratamiento con antibióticos. ${ }^{27}$

\section{Conclusiones}

Se presenta la experiencia clínica resultado de una investigación cuyo objetivo fue describir el comportamiento de la sepsis asociada a quimioterapia. La edad media de los pacientes fue 53 años y predominó el sexo masculino. El diagnóstico oncológico más frecuente fueron los tumores de vías digestivas. Del total de pacientes que desarrollaron eventos de infección asociada a la quimioterapia, la neutropenia febril fue el diagnóstisco más frecuente. No existió asociación significativa entre tipo de infección y el diagnóstico oncológico ni los esquemas de quimioterapia administrados. Es de imprescindible valor, realizar estudios descriptivos del comportamiento de las infecciones en los pacientes oncológicos en quimioterapia para poder brindar una atención médica cada vez más personalizada y eficaz.

\section{Bibliografía}

1. Santos SLV, Sousab TK, Costa DM et al. Infecciones asociadas a la atención de salud en un Hospital de Oncología Brasileño: análisis de cinco años. Enferm. glob. [Internet]. 2012;11(25):8-17. Disponible en: http:// scielo.isciii.es/scielo.php?script=sci_arttext\&pid=S1695-61412012000100002\&l$\mathrm{ng}=\mathrm{es} . \mathrm{http}: / / \mathrm{dx} . \mathrm{doi}$.org/10.4321/ S1695-61412012000100002. [Citado 6 de diciembre 2019].

2. Maldonado MS. Infecciones en el paciente oncológico. Revista Española pediatria 2013; 69(3):140-54.

3. Kontoyiannis DP. Prevention of fungal disease in chronically immunosuppressed outpatients: time for action? Ann Intern Med. 2013;158: 555-6.

4. Gea-Banacloche JC. Rituximab-associated infections. SeminHematol 2017; 47:187-98. 
5. Via y Rada F, Vega J, Torres C et al. Evolución de la neutropenia febril en pacientes con neoplasias sólidas y linfoproliferativas, 2015;5(1).

6. Cortés-Funes $\mathrm{H}$, Colomer $\mathrm{R}$ et al. Tratado de Oncología. P. Permanyer 2019; 2(86):887-90.

7. Dillman R. Cancer Immunotherapy. Cancer biotherapy and radiopharmaceuticals. 2011; 26(1). Disponible en https://doi.org/10.1089/ cbr.2010.0902.

8. Beyar-Katz O, Dickstein Y, Borok S, Vidal L, Leibovici L, Paul M. Empirical antibiotics targeting gram-positive bacteria for the treatment of febrile neutropenic patients with cancer. Cochrane Database Syst Rev. 2017; 6(6):CD003914. Doi: 10.1002/14651858. CD003914.pub4. PMID: 28577308; PMCID: PMC6481386.

9. Seo S, Campbell AP, Xie $\mathrm{H}$ et al. Outcome of respiratory syncytial virus lower respiratory tract disease in hematopoietic cell transplant recipients receiving aerosolized ribavirin: significance of stem cell source and oxygen requirement. Biol Blood Marrow Transplant 2018;19:589-96.

10. Ray CEJ, Lorenz JM, Burke CT et al. ACR Appropriateness Criteria radiologic management of benign and malignant biliary obstruction. J Am CollRadiol 2013; 10:567-74.

11. Borga G, Schloeter J, Rey. Hemocultivo positivo como factor pronóstico en pacientes con patología oncológica y neutropenia febril. Med Interna (Caracas) 2014;30(4):217-28.

12. Schelenz S, Giles D, Abdallah S. Epidemiology, management and economic impact of febrile neutropenia in oncology patients receiving routine care at a regional UK cancer centre. Ann Oncol. 2012;23(7):1889-93. Doi: 10.1093/annonc/mdr520. Epub 2011 Nov 2. PMID: 22048152.
13. Bardossy AC, Petiti HG, Neutropenia febril: agentes etiologicos y respuesta a antibioticoterapia empírica en el Hospital Privado S.ACentro Médico de Córdoba durante 2006-2007 Experiencia Médica, 2011;29(1).

14. Escobar Y, Blasco A, Espinosa J et al. Manual SEOM de cuidados continuos; (2da ed.). 2014; 163-166.

15. 14.García T, Pascual FJ, Ruiz M et al. Análisis de la eficacia diagnóstica de los hemocultivos en pacientes oncológicos. Revista Calidad Asistencial. 2016; 28(3):193-4. Disponible en http:// dx.doi.org/10.1016/j.cali.2012.09.006.

16. Kern WV, Marchetti O, Drgona L et al. Oral antibiotics for fever in low-risk neutropenic patients with cancer: a double-blind, randomized, multicenter trial comparing single daily moxifloxacin with twice daily ciprofloxacin plus amoxicillin/clavulanic acid combination therapy-EORTC infectious diseases group trial XV. J ClinOncol, 2013;31:1149-56.

17. Stoll P. Effectiveness of a protective environment implementation for cancer patients with chemotherapy-induced neutropenia on fever and mortality incidence. Am J Infec Control, 2016;41:357-9.

18. Pérez N, Cruz Y, Moya G et al. Caracterización estructural del factor estimulador de colonias de los granulocitos, Hebervital. VacciMonitor 2014; 23(1):3-10.

19. Bohlius J, Herbst C, Reiser M, Schwarzer G, Engert A. Granulopoiesis-stimulating factors to prevent adverse effects in the treatment of malignant lymphoma. Cochrane Database Syst Rev. 2008;2018(4):CD003189. Doi: 10.1002/14651858.CD003189.pub4. PMID: 18843642; PMCID: PMC7144686.

60 | Ciencia y Salud 2020; 4(3, septiembre-diciembre): Casos clínicos • 53-61 
20. Cooper KL, Madan J, Whyte S et al. Granulocyte colony-stimulating factors for febrile neutropenia prophylaxis following chemotherapy: systematic review and meta-analysis. BMC Cancer 2011;11:404.

21. Adrover E, Alba E, Alonso MA et al. Manual SEOM de Cuidados Continuos, (2da ed.). Sociedad Española de Oncología Médica. 2018; 137-141.

22. Klastersky J, Paesmans M. The Multinational Association for Supportive Care in Cancer (MASCC) risk index score: 10 years of use for identifying low-risk febrile neutropenic cancer patients. Support Care Cancer. 2013;21(5):148795. Doi: 10.1007/s00520-013-1758-y. Epub 2013 Feb 27. PMID: 23443617.

23. Marín M, GudiolC, García-Vidal Cetal. Bloodstream infections in patients with solid tumors: Epidemiology, antibiotic therapy, and outcomes in 528 episodes in a single cancer center. Medicine 2014; 93:143-9; Disponible en http:// dx.doi.org/10.1097/MD.0000000000000026.

24. 23. Via y Rada F, Vega J, Torres C et al. Evolución de la neutropenia febril en pacientes con neoplasias sólidas y linfoproliferativas. 2015;5(1).
25. Aguado JM, Cruz JJ, Viruzuela JA et al. Manejo de la infección y la neutropenia febril en el paciente con cáncer sólido. Enfermedades Infecciosas y Microbiología Clínica, 2017;35(7). Doi: 10.1016/j.eimc.2015.06.005.

26. Vidal L, Ben Dor I, Paul M et al. Oral versus intravenous antibiotic treatment for febrile neutropenia in cancer patients. Cochrane Database Syst Rev. 2015;10: CD003992. Doi: 10.1002/14651858.CD003992.pub3. PMID: 24105485 ; PMCID: PMC6457615.

27. Velázquez I, Aranda J, Camacho JL et al. Epidemiología de infecciones nosocomiales en el Instituto Jalisciense de Cancerología. Rev Cubana Salud Pública 2013;39(1). Versión ISSN 0864-3466. Disponible en: http:// scielo.sld.cu/scielo.php?script=sci_arttext \&pi$\mathrm{d}=$ S0864-34662013000100003\&lng=es.

28. NCCN Clinical Practice Guidelines in Oncology (NCCN Guidelines). Prevention and treatment of cancer-related infections. Version $1 ; 2019$. 